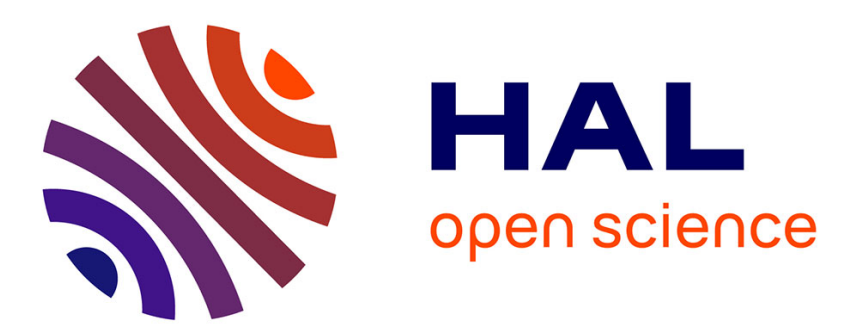

\title{
A two level non linear inverse control structure for rotorcraft trajectory tracking \\ Felix Mora-Camino
}

\section{To cite this version:}

Felix Mora-Camino. A two level non linear inverse control structure for rotorcraft trajectory tracking. CCC 2007, 26th Chinese Control Conference, Jul 2007, Zhangjiajie, China. pp 321-325, 10.1109/CHICC.2006.4347095 . hal-00938173

\section{HAL Id: hal-00938173 \\ https://hal-enac.archives-ouvertes.fr/hal-00938173}

Submitted on 23 Jun 2014

HAL is a multi-disciplinary open access archive for the deposit and dissemination of scientific research documents, whether they are published or not. The documents may come from teaching and research institutions in France or abroad, or from public or private research centers.
L'archive ouverte pluridisciplinaire HAL, est destinée au dépôt et à la diffusion de documents scientifiques de niveau recherche, publiés ou non, émanant des établissements d'enseignement et de recherche français ou étrangers, des laboratoires publics ou privés. 


\title{
A Two Level Non Linear Inverse Control Structure for Rotorcraft Trajectory Tracking
}

\author{
Mora-Camino Félix \\ LARA ENAC, 31055 Toulouse, France \\ E-mail: felix.mora@enac.fr
}

\begin{abstract}
The purpose of this communication is to investigate the usefulness of the non linear inverse control approach to solve the trajectory tracking problem for a four rotor aircraft. After introducing simplifying assumptions, the flight dynamics equations for the four rotor aircraft are considered. A trajectory tracking control structure based on a two layer non linear inverse approach is then proposed. A supervision level is introduced to take into account the actuator limitations.
\end{abstract}

Key Words: Rotorcraft flight mechanics, nonlinear inverse control, trajectory tracking.

\section{INTRODUCTION}

In the last years a large interest has risen for the four rotor concept since it appears to present simultaneously hovering, orientation and trajectory tracking capabilities of interest in many practical applications ${ }^{[1]}$. The flight mechanics of rotorcraft are highly non linear and different control approaches (integral LQR techniques, integral sliding mode control ${ }^{[2]}$, reinforcement learning $^{[3]}$ ) have been considered with little success to achieve not only autonomous hovering and orientation, but also trajectory tracking In this paper, after introducing some simplifying assumptions, the flight dynamics equations for a four rotor aircraft with fixed pitch blades are considered.

The purpose of this study is to investigate the usefulness of the non linear inverse control approach to solve the trajectory tracking problem for this class of rotorcraft. This approach has been already considered in the case of aircraft trajectory tracking by different authors ${ }^{[4-6]}$. It appears that the flight dynamics of the considered rotorcraft present a two level input affine structure which is made apparent when a new set of equivalent inputs is defined. This allows to introduce a non linear inverse control approach with two time scales, one devoted to attitude control and one devoted to orientation and trajectory tracking.

\section{FLIGHT DYNAMICS EQUATIONS OF ROTORCRAFT}

The considered system is shown in Fig. 1 where rotors one and three are clockwise while rotors two and four are counter clockwise. Annex 1 describes the rotor dynamics.

The main simplifying assumptions adopted with respect to flight dynamics in this study are a rigid cross structure, no wind, negligible aerodynamic contributions resulting from translational speed, no ground effect as well as negligible air density effects and very small rotor response times. It is then possible to write simplified rotorcraft flight equations ${ }^{[7]}$.

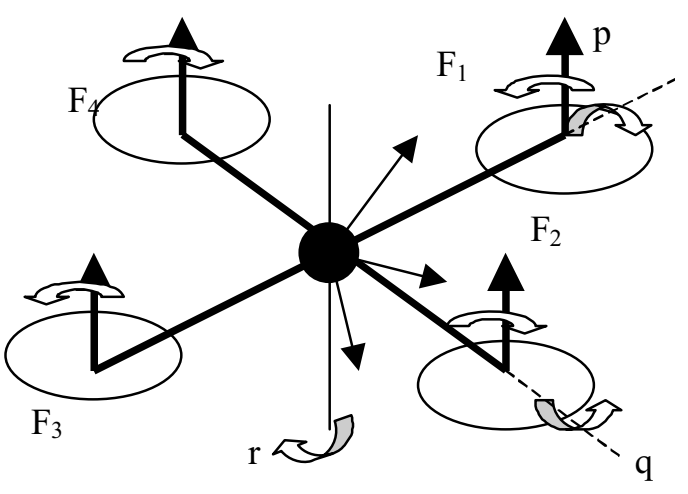

Fig. 1 Four rotor aircraft

The moment equations can be written as:

$$
\left.\begin{array}{l}
\dot{p}=\left(a / I_{x x}\right)\left(F_{4}-F_{2}\right)+k_{2} q r \\
\dot{q}=\left(a / I_{y y}\right)\left(F_{1}-F_{3}\right)+k_{4} p r \\
\dot{r}=\left(k / I_{z z}\right)\left(F_{2}-F_{1}+F_{4}-F_{3}\right)
\end{array}\right\}
$$

where $p, q, r$ are the components of the body angular velocity, with $k_{2}\left(I_{z z}-I_{y v}\right) / I_{x x} \quad$ and $k_{4}=\left(I_{x x}-I_{z z}\right) / I_{y y}, I_{x x}, I_{y y}$ and $I_{z z}$ being the moments of inertia in body-axis and $m$ the total mass of the rotorcraft.

The Euler equations are given by:

$$
\begin{aligned}
& \dot{\phi}=p+\tan (\theta) \sin (\phi) q+\tan (\theta) \cos (\phi) r \\
& \dot{\theta}=\cos (\phi) q-\sin (\phi) r \\
& \dot{\psi}=((\sin (\phi) / \cos (\theta)) q+(\cos (\phi) / \cos (\theta)) r
\end{aligned}
$$

where $\theta, \phi$, and $\psi$ are respectively the pitch, bank and heading angles.

The acceleration equations written directly in the local Earth reference system are such as:

$$
\left.\begin{array}{l}
\ddot{x}=(1 / m)(\cos (\psi) \sin (\theta) \cos (\phi)+\sin (\psi) \sin (\phi)) F \\
\ddot{y}=(1 / m)(\sin (\psi) \sin (\theta) \cos (\phi)-\cos (\psi) \sin (\phi)) F \\
\ddot{z}=-g+(1 / M) \cos (\theta) \cos (\phi) F
\end{array}\right\}
$$

where $x, y$ and $z$ are the centre of gravity coordinates

and where :

$$
F=F_{1}+F_{2}+F_{3}+F_{4}
$$

and with the constraints: 


$$
0 \leqslant F_{i_{i}} \leqslant F_{\text {max }} \quad i \in\{1,2,3,4\}
$$

\section{THE PROPOSED FLIGHT CONTROL APPROACH}

Here we are interested in controlling the four rotor aircraft so that its centre of gravity follows a given path with a given heading $\psi$ while attitude angles $\theta$ and $\phi$ remain small. Many potential applications require not only the centre of gravity of the device to follow a given trajectory but also the aircraft to present a given orientation. From equations (1) it appears that the effectiveness of the rotor actuators is much larger with respect to the roll and pitch axis than with respect to the yaw axis. Then we consider that attitude control is involved with controlling the $\theta$ and $\phi$ angles. In equations (1) the effect of rotor forces appears as differences so, we define new attitude inputs $u_{1}$ and $u_{2}$ as:

$$
u_{1}=F_{1}-F_{3}, \quad u_{2}=F_{2}-F_{4}
$$

In the heading and position dynamics, the effects of rotor forces and moments appear as sums, so we define new guidance inputs $v_{1}$ and $v_{2}$ as:

$$
v_{1}=F_{1}+F_{3}, \quad v_{2}=F_{2}+F_{4}
$$

It is supposed that $u_{1}$ and $u_{2}$ can be made to vary significantly while $v_{l}$ and $v_{2}$ can remain constant.

\subsection{Attitude Control Layer}

Then the attitude dynamics can be rewritten under the affine input form:

with

$$
\begin{gathered}
\dot{\dot{X}}=f(\underline{X}, \underline{V})+g(\underline{X}) \underline{U} \\
\underline{Y}^{\prime}=(\theta, \phi)
\end{gathered}
$$

$$
\begin{gathered}
\underline{X}^{\prime}=(p, q, \theta, \phi), \underline{U}^{\prime}=\left(u_{1}, u_{2}\right) \\
\text { and } \underline{V}^{\prime}=\left(v_{1}, v_{2}\right)
\end{gathered}
$$

Then, considering the non linear inverse control theory, it appears that the attitude angles present relative degrees equal to one and that there is no internal dynamics while the output equations can be rewritten as:

$$
\underline{\ddot{Y}}=M(\underline{Y}) \underline{U}+N(\underline{X}) \underline{V}+P(\underline{X})
$$

with

$$
\begin{gathered}
M(\underline{Y})=\left[\begin{array}{cc}
a \cos \phi / I_{y y} & 0 \\
a \tan \theta \sin \phi / I_{y y} & -a / I_{x x}
\end{array}\right] \\
N(\underline{X})=\left[\begin{array}{cc}
k \sin \phi / I_{z z} & -k \sin \phi / I_{z z} \\
-k \tan \theta \cos \phi / I_{z z} & k \tan \theta \cos \phi / I_{z z}
\end{array}\right] \\
P(\underline{X})=\left[P_{1}, P_{2}\right]^{\prime}
\end{gathered}
$$

where:

$$
\begin{gathered}
P_{1}=k_{4} \cos \phi p r \\
-(q \sin \phi+r \cos \phi)(p+\tan \theta(q \sin \phi+r \tan \theta \cos \phi)) \\
P_{2}=k_{2} q r+k_{4} p r \tan \theta \sin \phi \\
+q \mathrm{~d}(\tan \theta \sin \phi) / \mathrm{dt}+r \mathrm{~d}(\tan \theta \cos \phi) / \mathrm{d} t
\end{gathered}
$$

It appears that while $\phi \neq \pm \pi / 2$, the attitude dynamics given by (9) are invertible. Then it appears feasible to consider as control objective to get second order linear attitude dynamics towards reference values:

$$
\left[\begin{array}{c}
\ddot{\theta}_{d} \\
\ddot{\phi}_{d}
\end{array}\right]=\underline{Y}_{d}=\left[\begin{array}{l}
-2 \zeta_{\theta} \omega_{\theta} \dot{\theta}-\omega_{\theta}{ }^{2}\left(\theta-\theta_{c}\right) \\
-2 \zeta_{\phi} \omega_{\phi} \dot{\phi}-\omega_{\phi}{ }^{2}\left(\phi-\phi_{c}\right)
\end{array}\right]
$$

where $\zeta_{\theta}, \zeta_{\phi}, \omega_{\theta}, \omega_{\phi}$ are respectively damping and natural frequency parameters while $\theta_{c}$ and $\phi_{c}$ are reference values for the attitude angles.

Then the corresponding non linear inverse attitude control law is given by:

$$
\underline{U}=-M(\underline{Y})^{-1}\left(N(\underline{X}) \underline{V}+P(\underline{X})-\underline{\ddot{Y}}_{d}\right)
$$

\subsection{Guidance Control Layer}

Considering that the attitude dynamics are stable and faster than the guidance dynamics, the guidance equations can be approximated by the control affine form:

$$
\left[\begin{array}{c}
\ddot{\psi} \\
\ddot{x} \\
\ddot{y} \\
\ddot{z}
\end{array}\right]=\left[\begin{array}{c}
\frac{k \cos \phi_{c}}{I_{z} \cos \theta_{c}}\left(v_{2}-v_{1}\right) \\
(1 / m)\left(\cos (\psi) \sin \left(\theta_{c}\right) \cos \left(\phi_{c}\right)+\sin (\psi) \sin \left(\phi_{c}\right)\right)\left(v_{1}+v_{2}\right) \\
(1 / m)\left(\sin (\psi) \sin \left(\theta_{c}\right) \cos \left(\phi_{c}\right)-\cos (\psi) \sin \left(\phi_{c}\right)\right)\left(v_{1}+v_{2}\right) \\
-g+(1 / m) \cos \left(\theta_{c}\right) \cos \left(\phi_{c}\right)\left(v_{1}+v_{2}\right)
\end{array}\right]
$$

Here also, the outputs of the guidance dynamics present relative degrees equal to 1 while the internal dynamics, which are concerned with the attitude angles, are supposed already stabilized. Then, supposing that second order linear dynamics are of interest for the guidance variables, we can define desired accelerations by:

$$
\left[\begin{array}{l}
\ddot{\psi}_{c} \\
\ddot{x}_{c} \\
\ddot{y}_{c} \\
\ddot{z}_{c}
\end{array}\right]=\left[\begin{array}{c}
-2 \zeta_{\psi} \omega_{\psi} \dot{\psi}-\omega_{\psi}{ }^{2}\left(\psi-\psi_{c}\right) \\
-2 \zeta_{x} \omega_{x} \dot{x}-\omega_{x}{ }^{2}\left(x-x_{c}\right) \\
-2 \zeta_{y} \omega_{y} \dot{y}-\omega_{y}{ }^{2}\left(y-y_{c}\right) \\
-2 \zeta_{z} \omega_{z} \dot{z}-\omega_{z}{ }^{2}\left(z-z_{c}\right)
\end{array}\right]
$$

where $\zeta_{\psi}, \zeta_{x}, \zeta_{y}, \zeta_{z}, \omega_{\psi}, \omega_{x}, \omega_{y}, \omega_{z}$ are respectively damping and natural frequency parameters while $\psi_{c}, x_{c}$, $y_{c}$ and $z_{c}$ are reference values for the attitude angles. Of course, many other schemes can be proposed to define desired accelerations at the guidance level.

Once desired accelerations are made available, the inversion of the guidance dynamics brings nominal the solution:

$$
\begin{aligned}
& v_{1}=\left(\frac{1}{2} m \sqrt{\ddot{x}_{c}^{2}+\ddot{y}_{c}^{2}+\left(\ddot{z}_{c}+g\right)^{2}}-\frac{I_{z z} \cos \theta_{c}}{k \cos \phi_{c}} \ddot{\psi}_{c}\right) \\
& v_{2}=\left(\frac{1}{2} m \sqrt{\ddot{x}_{c}^{2}+\ddot{y}_{c}^{2}+\left(\ddot{z}_{c}+g\right)^{2}}+\frac{I_{z z} \cos \theta_{c}}{k \cos \phi_{c}} \ddot{\psi}_{c}\right)
\end{aligned}
$$

with

$$
\theta_{c}=\arctan \left(\left(\ddot{x}_{c} \cos \psi+\ddot{y}_{c} \sin \psi\right) /\left(\ddot{z}_{c}+g\right)\right)
$$

and

$$
\phi_{c}=\arcsin \left(\frac{\ddot{x}_{c} \sin \psi-\ddot{y}_{d} \cos \psi}{\sqrt{\ddot{x}_{d}{ }^{2}+\ddot{y}_{d}{ }^{2}+\left(\ddot{z}_{d}+g\right)^{2}}}\right.
$$




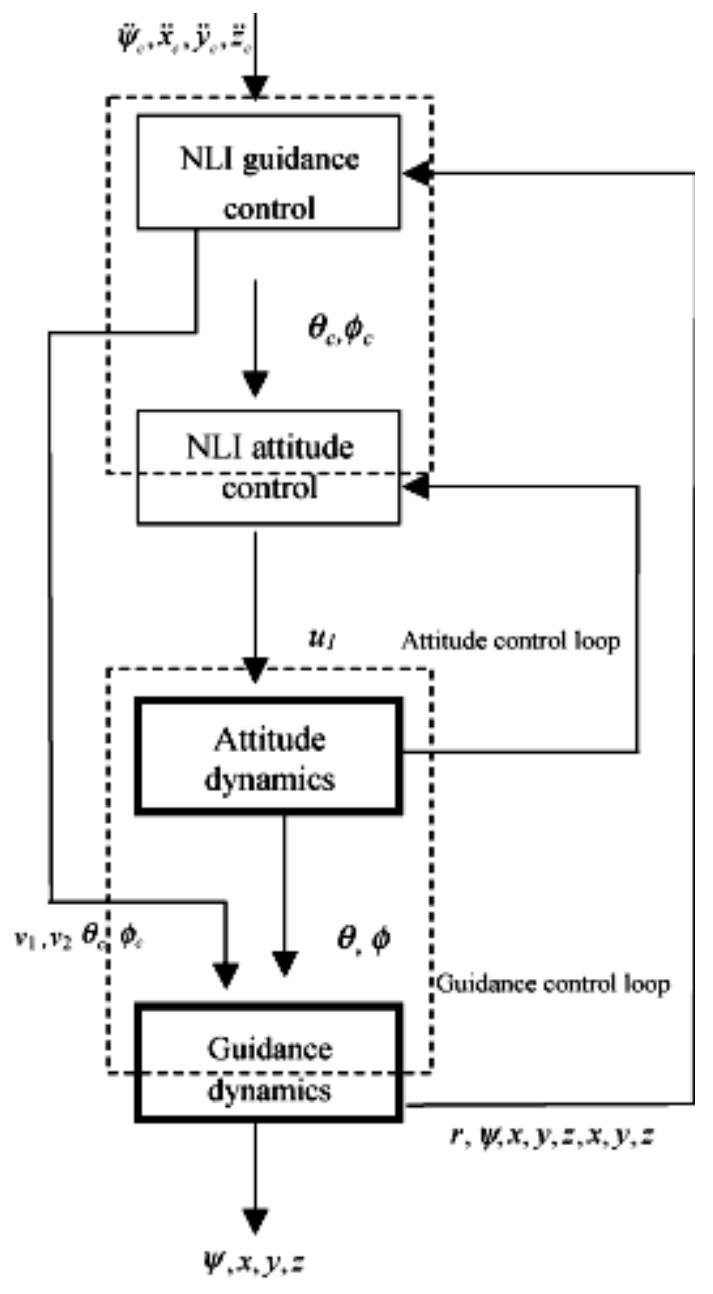

Fig. 2 Proposed control structure

Then, returning to the expression of the attitude control law, it happens that the centre of gravity acceleration terms compensate each others and the law becomes:

$$
\underline{U}=-M(\underline{Y})^{-1}\left(\underline{N}(\underline{X})+P(\underline{X})-\underline{\ddot{Y}}_{d}\right)
$$

with

$$
\underline{N}(\underline{X})=\left[\begin{array}{c}
N_{1} \\
N_{2}
\end{array}\right]=\left[\begin{array}{c}
-\cos \theta_{c} \frac{\sin \phi}{\cos \phi_{c}} \\
\sin \theta \frac{\cos \theta_{c}}{\cos \theta} \frac{\cos \phi}{\cos \phi_{c}}
\end{array}\right] \ddot{\psi}_{c}
$$

The whole proposed control structure is given in Fig. 2.

\section{FLIGHT CONTROL SUPERVISION}

Since the above control approach does not consider explicitly the input level constraints, we introduce here a supervision layer whose function is to avoid the generation of unfeasible reference values for the inputs by modifying, as less as possible, the current control objectives. According to (5), (6) and (7), the control signals should be such as:

$$
-F_{\text {max }} \leqslant u_{i} \leqslant F_{\text {max }}, i=1,2
$$

and

$$
0 \leqslant v_{i} \leqslant 2 F_{\max }, i=1,2
$$

Conditions (21a) implies for the desired attitude accelerations to satisfy the following conditions: with

$$
\ddot{\phi}_{\min } \leqslant \ddot{\phi}_{c} \leqslant \ddot{\phi}_{\max }
$$

$$
\ddot{\phi}_{\text {min }}=N_{2}+P_{2}-\frac{a \cos \phi}{I_{y y}} F_{\text {max }}
$$

and

$$
\ddot{\phi}_{\max }=N_{2}+P_{2}+\frac{a \cos \phi}{I_{y y}} F_{\text {max }}
$$

and condition:

with

$$
\ddot{\theta}_{\min } \leqslant \ddot{\theta}_{c}-\tan \theta \ddot{\phi}_{c} \leqslant \ddot{\theta}_{\text {max }}
$$

and

$$
\ddot{\theta}_{\text {max }}=a F_{\text {max }} / I_{x x}+\left(N_{1}+P_{1}\right)-\tan \theta\left(N_{2}+P_{2}\right)
$$

Then, reference values for instant attitude angles accelerations can be obtained from the solution of the following linear-quadratic optimization problem:

$$
\min _{\alpha_{r} \beta}\left(\ddot{\theta}_{c}-\alpha\right)^{2}+\left(\ddot{\phi_{c}}-\beta\right)^{2}
$$

with

$$
\begin{gathered}
\ddot{\phi}_{\min } \leqslant \beta \leqslant \ddot{\phi}_{\max } \\
\ddot{\theta}_{\min } \leqslant \alpha-\tan \theta \beta \leqslant \ddot{\theta}_{\text {max }}
\end{gathered}
$$

Observe that the solution of this problem is equal to $\left(\ddot{\theta}_{c}, \ddot{\phi}_{c}\right)$ if it is feasible with respect to constraints $(24 \mathrm{~b})$ and (24c), otherwise the solution will be on the border of the convex feasible set.

Then if $\alpha^{*}$ and $\beta^{*}$ are solution of this problem, $u_{1}$ and $u_{2}$ are given by:

$$
\left[\begin{array}{l}
u_{1} \\
u_{2}
\end{array}\right]=-M(\underline{Y})^{-1}\left(N(\underline{X}) \underline{V}+P(\underline{X})-\left[\begin{array}{l}
\alpha^{*} \\
\beta^{*}
\end{array}\right]\right)
$$

In the case of $v_{1}$ and $v_{2}$ (relations (21b)) and considering the expressions of $\theta_{c}$ and $\phi_{c}$ the above approach leads to the consideration of an intricate non convex optimization problem. A different approach is proposed here. Let $\lambda$ be such as:

$$
\ddot{x}_{r}=\lambda \ddot{x}_{c}, \ddot{y}_{r}=\lambda \ddot{y}_{c}, \ddot{z}_{r}+g=\lambda\left(\ddot{z}_{c}+g\right)
$$

then according to (18a) and (18b):

$$
\theta_{r}=\theta_{c} \quad \text { and } \quad \phi_{r}=\phi_{c}
$$

Feasible reference values for $\ddot{x}_{r}, \ddot{y}_{r}, \ddot{z}_{r}$ and $\ddot{\psi}_{r}$ can be obtained from the solution of the following linear -quadratic optimization problem:

$$
\min _{\lambda, \mu}(\lambda-1)^{2}+\eta^{2}\left(\mu-\ddot{\psi}_{c}\right)^{2}
$$

with

$$
\begin{aligned}
& 0 \leqslant\left(m \sqrt{\ddot{x}_{c}^{2}+\ddot{y}_{c}^{2}+\left(\ddot{z}_{c}+g\right)^{2}}\right) \lambda-\left(\frac{I_{z z} \cos \theta_{c}}{k \cos \phi_{c}}\right) \mu \leqslant 4 F_{\text {max }} \\
& 0 \leqslant\left(m \sqrt{\ddot{x}_{c}^{2}+\ddot{y}_{c}^{2}+\left(\ddot{z}_{c}+g\right)^{2}}\right) \lambda+\left(\frac{I_{z z} \cos \theta_{c}}{k \cos \phi_{c}}\right) \mu \leqslant 4 F_{\text {max }}
\end{aligned}
$$

where $\eta$ is here a time constant. Let $\lambda^{*}$ and $\mu^{*}$ be the solution of the above problem, then the control inputs can be taken as: 


$$
\begin{aligned}
& v_{1}=\frac{1}{2}\left(m \lambda^{*} \sqrt{\ddot{x}_{c}^{2}+\ddot{y}_{c}^{2}+\left(\ddot{z}_{c}+g\right)^{2}}-\frac{I_{z z} \cos \theta_{c}}{k \cos \phi_{c}} \mu^{*}\right) \\
& v_{2}=\frac{1}{2}\left(m \lambda^{*} \sqrt{\ddot{x}_{c}^{2}+\ddot{y}_{c}^{2}+\left(\ddot{z}_{c}+g\right)^{2}}+\frac{I_{z z} \cos \theta_{c}}{k \cos \phi_{c}} \mu^{*}\right)
\end{aligned}
$$

Then:

$$
\begin{array}{ll}
F_{1}=\left(u_{1}+v_{1}\right) / 2, & F_{2}=\left(u_{2}+v_{2}\right) / 2 \\
F_{3}=\left(v_{1}-u_{1}\right) / 2, & F_{4}=\left(v_{2}-u_{2}\right) / 2
\end{array}
$$

\section{CASE STUDIES}

Here we consider two cases:

One where the objective is to hover at an initial position of coordinates $x_{0}, y_{0}, z_{0}$ while acquiring a new orientation $\psi_{1}$, and

One where the rotorcraft is tracking the helicoïdal trajectory of equations:

$$
\left.\begin{array}{c}
x_{c}(t)=\rho \cos v t \\
y_{c}(t)=\rho \sin v t \\
z_{c}=\delta+\gamma t \\
\psi_{c}(t)=v t+\pi / 2
\end{array}\right\}
$$

where $\rho$ is a constant radius and $\gamma$ is a constant path angle.

\subsection{Heading Control at Hover}

In this case we get the guidance control laws:

$$
\begin{aligned}
& v_{1}=\frac{1}{2}\left(m g-\frac{I_{z z}}{k} \ddot{\psi}_{c}\right) \\
& v_{2}=\frac{1}{2}\left(m g+\frac{I_{z z}}{k} \ddot{\psi}_{c}\right)
\end{aligned}
$$

with the following reference values for the attitude angles:

$$
\theta_{c}=0 \quad \text { and } \quad \phi_{c}=0
$$

Here the heading acceleration is given by:

$$
\ddot{\psi}_{c}=-2 \zeta_{\psi} \omega_{\psi} r-\omega_{\psi}{ }^{2}\left(\psi-\psi_{1}\right)
$$

Starting from an horizontal attitude $(\theta(0)=0, \phi(0)=0)$, attitude inputs $u_{1}$ and $u_{2}$ given by relation (14) remain equal to zero. Then, Fig. 3 and 4 display some simulation results:

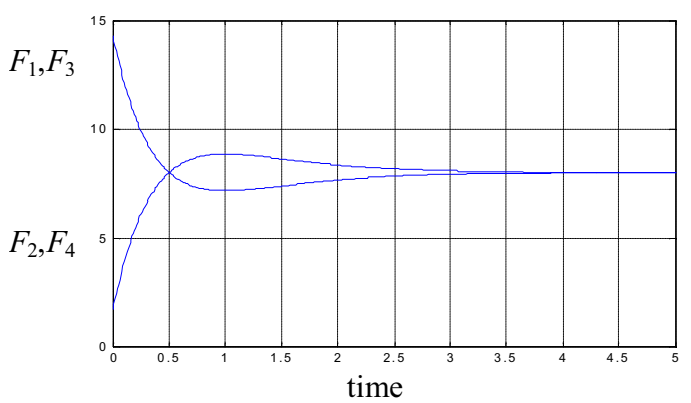

Fig. 3 Hover control inputs

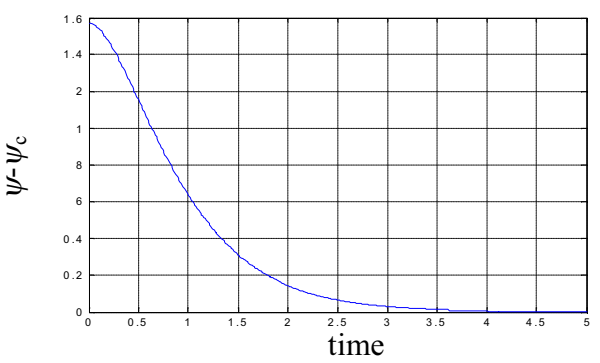

Fig. 4 Heading response during hover

\subsection{Trajectory Tracking Case}

In this case we get the guidance control laws:

$$
v_{1}=v_{2}=\frac{1}{2}\left(m \sqrt{\rho^{2} v^{2}+g^{2}}\right)
$$

Here the permanent reference values for the attitude angles are such as:

$$
\theta_{c}=0
$$

and

$$
\sin \phi_{c}=-\frac{\rho v^{2}}{\sqrt{\rho^{2} v^{4}+g^{2}}}
$$

and the desired guidance and orientation accelerations are given by:

$$
\left.\begin{array}{c}
\ddot{x}_{c}=-\rho v^{2} \cos (v t) \\
\ddot{y}_{c}=-\rho v^{2} \sin (v t) \\
\ddot{z}_{c}=0, \quad \ddot{\psi}_{c}=0
\end{array}\right\}
$$

Attitude inputs are given by relation (14) where now:

$$
M^{-1}=\left[\begin{array}{cc}
0 & I_{y y} /(a \cos \phi) \\
-1 /\left(a I_{x x}\right) & \tan \theta \tan \phi /\left(a I_{x x}\right)
\end{array}\right]
$$

and

$$
\underline{N}(\underline{X})^{\prime}=\left[\begin{array}{ll}
0 & 0
\end{array}\right]
$$

In Fig. $5 \sim 7$ simulation results are displayed where at initial time the rotorcraft is hovering:

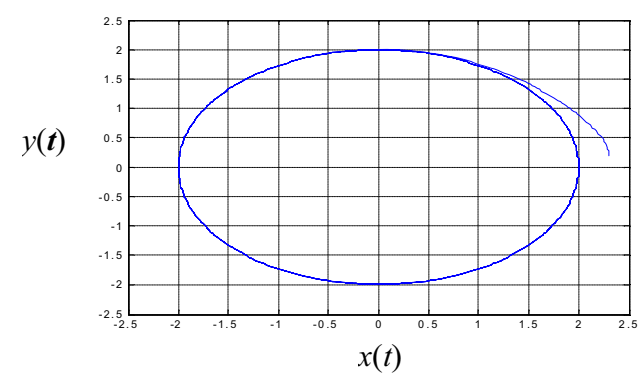

Fig. 5 Evolution of rotorcraft horizontal track

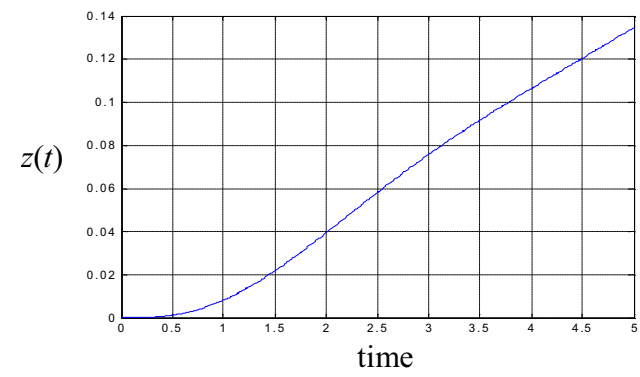

Fig. 6 Evolution of rotorcraft altitude 


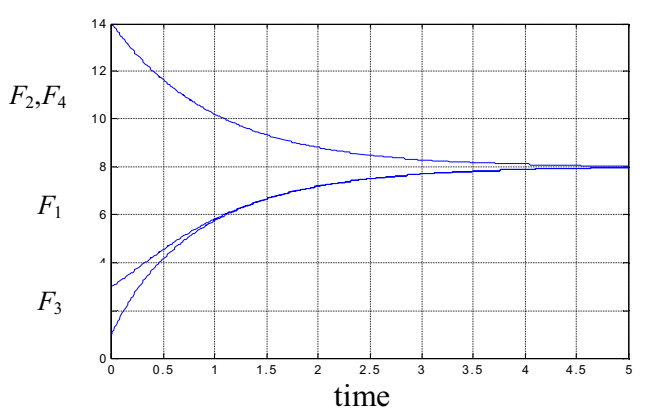

Fig. 7 Rotorcraft trajectory tracking inputs

\section{CONCLUSIONS}

In this communication the theoretical applicability of the non linear inverse control technique to rotorcraft trajectory tracking has been investigated. It appears that this approach leads to the design of a two level control structure based on analytical laws. Considering the structure of the rotorcraft flight dynamics, other promising non linear control techniques are differential flat control $^{[8]}$ and back stepping control ${ }^{[9]}$.

When considering the complexity of these non linear control laws involving a relatively small number of inputs, neural networks components could be of interest for their effective implementation.

However, the robustness of these control laws with respect to the different aerodynamic effects which have been taken as negligible should be investigated. Since only very intricate theories are available to approach this problem, real flight tests appear, at this stage, to be un- avoidable.

\section{REFERENCES}

[1] Hoffmann G, Rajnarayan D G, Waslander S L, Dostal D, Jang J S, Tomlin, C J. The Standford Tetsbed of Autonomous Rotorcraft for Multi-Agent Control// $23^{\text {rd }}$ Digital Avionics Systems Conference. Salt Lake City, UT, November 2004.

[2] Hassan K Khalil, Nonlinear Systems. 3rd Ed. Prentice Hall, 2002.

[3] Sutton R S, Barto A G. Reinforcement learning: an introduction. MA: Cambridge MIT Press, 1998.

[4] Singh S N, Schy A A. Nonlinear decoupled control synthesis for manoeuvring aircraft // Proceedings of the 1978 IEEE conference on Decision and Control, Piscataway, NJ, 1978.

[5] Ghosh R, TomlinC J. Nonlinear Inverse Dynamic Control for Model-based Flight. Proceeding of AIAA, 2000.

[6] Asep R, Shen T J, Achaíbou K, Mora-Camino F. An application of the nonlinear inverse technique to flight-path supervision and control// Proceedings of the 9th International Conference of Systems Engineering, Las Vegas,NV, 1993.

[7] Etkin B, Reid L R. Dynamics of Flight-Stability and Control. New York NY: John Wiley \& Sons, 1996.

[8] Lu W C, Mora-Camino F, Achaibou K. Flight Mechanics and Differential Flatness// Dincon 04, Proceedings of Dynamics and Control Conference. Ilha Solteira, Brazil, 2004: 830-839.

[9] Miquel T. Contribution à la synthèse de lois de guidage relatif, approche non linéaire[D]. Toulouse : Université Paul Sabatier, 2004. 